\title{
XX.
}

\section{Ein Fall von Spina bifida occulta mit congeni- taler lumbaler Hypertrichose, Pes varus und ,Mal perforant du pied“".}

(Aus der chirurgischen Klinik zu Zürich.)

Von Dr. Conrad Brunner, Secundararzt.

(Hierzu Taf. X.)

Seit Virchow's Entdeckung der Spina bifida occulta ${ }^{1}$ ) ist die Diagnose dieser seltenen Missbildung in 4 weiteren Fällen ${ }^{2}$ ) am Lebenden gestellt worden. Bei dem zuletzt beschriebenen dieser Fälle wurde die Diagnose durch die Autopsie bestätigt und es hat v. Recklinghausen, gestïtzt auf seinen anatomischen Befund in einer jüngst publicirten Arbeit die interessante Erage beantwortet, inwiefern die sacrale Hypertrichose genetisch von einer Spaltbildung der Wirbelsäule abhänge. „Jede günstige Gelegenheit", sagt v. Recklinghausen, „die Beobachtung alter und gar abgelaufener Fälle von Spina bifida vorzunehmen, wird wegen der mannichfachen Fragen, die sich jetzt darüber erheben, in der nächsten Zeit gewiss mit Eifer ergriffen und nenes Material zusammengetragen werden zur Stütze des Satzes, dass die äber der Wirbelsäule gelegene, circumscripte Hypertrichose oft, wenn nicht immer mit einer Wirbelspalte zusammen vorkomme." Eine solche, wenn auch nicht durch die Autopsie gesicherte Bestätigung dieses Satzes findet sich in der Beobachtung eines Patienten, der an hiesiger Klinik von Herrn Professor Krönlein behandelt wurde und dessen Krankergeschichte wir im Folgenden mitzutheilen uns erlauben.

1) Zeitschrift für Ethnologie. Bd. VII. 1875. S. 279.

2) Dieses Archiv. Bd. 105. Heft 2. S. 281. Untersuchungen über die Spina bifida von Prof. F. v. Recklinghausen. (Auch als Sonder-Abdruck Berlin 1886. Georg Reimer.) 


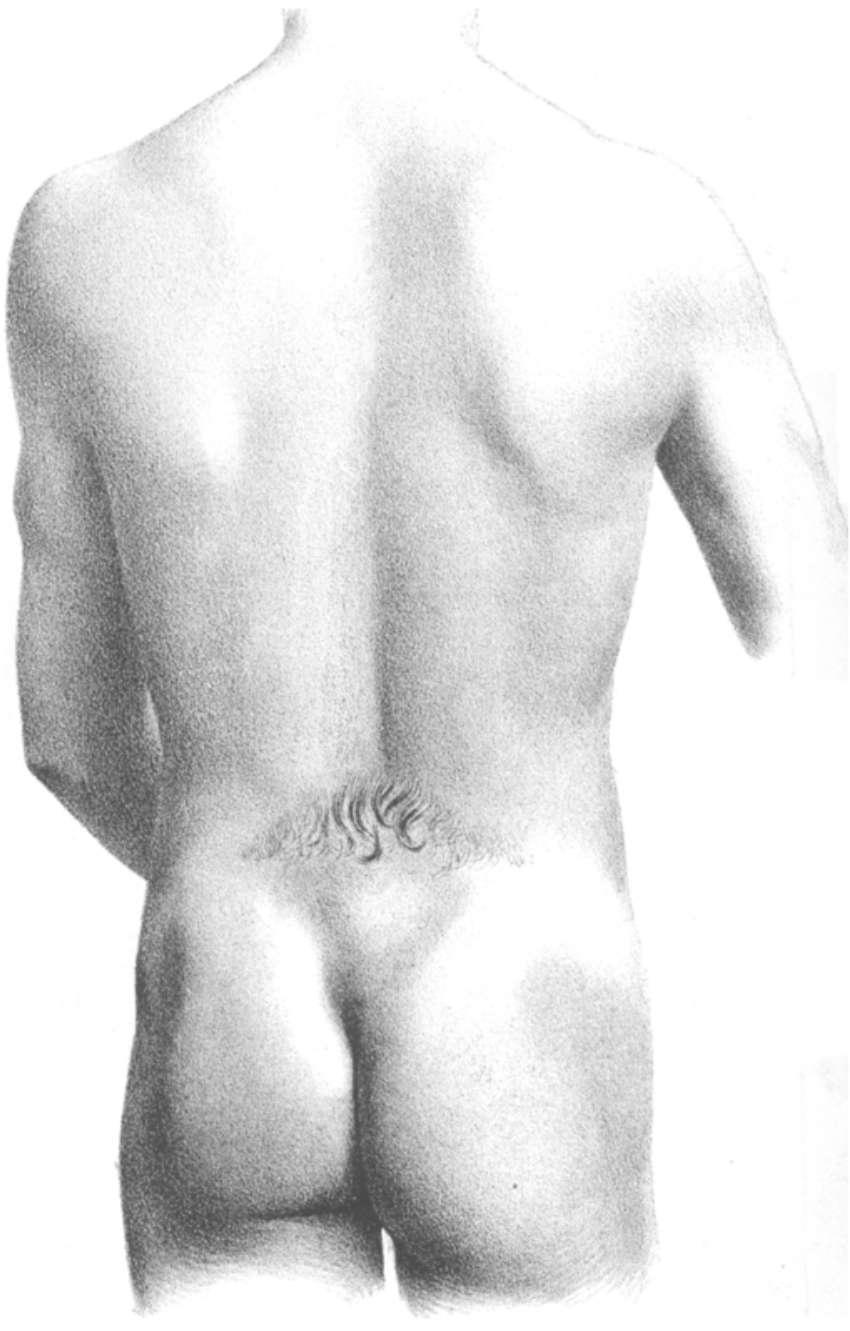


Anamnese. Amstein, Carl, Schnster von Willisau, 20 Jabre alt, trat am 11. Juni 1884 in hiesige Spitalbehandlung. Sein Vater starb an einer Krankheit, äber die Patient nichts Genaueres mittheilen kann. Die Mutter lebt und ist gesund. Von Missbildungen irgend welcher Art soll nichts in der Familie bekannt sein. Patient selbst war ausser dem unten zu beschreibenden Leiden stets gesund. Ueber Ursprung und Verlauf seiner Krankheit macht er folgende Angaben: Beide Füsse waren ursprünglich vollkommen gleich und wohl ausgebildet. Im Alter von 7 Jahren soll Patient beim Aebrenlesen auf eine Stoppel getreten sein und sich dadurch eine kleine Wunde im Kleinzehenballen des rechten Fusses zugezogen haben. Die Wunde blutete nur wenig, dagegen konnte Patient wegen der Schmerzen mehrere Tage nicht gehen. Unter Anwendung von Hausmitteln heilte die Wunde wieder zu; Patient konnte wieder herumgehen, doch will er stets etwas Scbmerz im rechten Fuss verspürt haben. 2 Jahre später bildete sich in der Gegend der früberen Verletzung eine kleine Geschwulst, die spontan aufbrach, wobei sich ausser Blut und Eiter ein Stück Strohhalm von $2 \mathrm{~cm}$ Länge entleerte. Die Perforationsöffnung heilte wieder zu, allein seitdem sollen immer von Zeit zu Zeit sich neue Aufbrüche, Eiterentleerungen und Ulcerationsprozesse eingestellt haben. Knochenstückchen sollen nie abgegangen sein. Ueber die Art, wie die Difformität des Fusses entstanden, lassen sich von dem Patienten keine genauen Aufschlüsse erhalten. Sicher scheint nur zu sein, dass der rechte Fuss vor dem Trauma in 7. Lebensjabr vollkommen normal gebildet war und es scheint demnach die Difformität das Endproduct der sich oft wiederholenden Dlcerationsprozesse zu sein. Seit jener Zeit soll das rechte Bein auch an Kraft abgenommen haben, es soll leichter ermüden und hie und da der Sitz zuckender Schmerzen sein. Zum letzten Mal erfolgte ein Aufbruch im Januar 1884, seither heilte die Wunde nicht mehr zu und Patient wandte sich deshalb an's hiesige Spital, woselbst er anfangs nur so lange blieb, bis die Wunde sich wieder schloss, worauf er nach Hause ging. Aber schon wenige Tage nach der Entlassung erfolgte ein neuer Aufbruch und da Patient desbalb geh- und auch arbeitsunfähig wurde, wünschte er amputirt zu werden.

In Bezug auf die unten beschriebene Haarlocke am Rücken des Patienten macht die Mutter desselben die Angabe, dass schon unmittelbar nach der Geburt an dieser Stelle eine Anzahl ziemlich langer, hellblonder Härchen vorhanden waren.

Status praesens. Der ziemlich intelligente Patient ist von kleiner Statur, dabei kräftig gebaut und gut genährt. Schädel brachycephal. Zähne ausgesprochen rachitisch. Hautcolorit hell. Hals kurz und dick; kleine Struma. Thorax schön geformt. Die Untersuchung der Brust- und Bauchorgane ergiebt normale VerhäItnisse. Im Urin kein Eiweiss. Die oberen Extremitäten weisen keinerlei Abnormitäten auf; dagegen ist die rechte untere Extremität, verglichen mit der linken, entschieden atropbisch und es weist ausserdem der linke Fuss eine unten genauer zu schildernde Verbildung auf. Yergleichende Messungen des rechten und linken Beines ergeben Folgendes: 


\section{6}

Die Länge des Ober- und Unterschenkels ist beiderseits gleich; in der Dickendimension hingegen ergeben sich folgende Unterschiede:

\begin{tabular}{|c|c|}
\hline Circumferenz & R. \\
\hline Mitte des Oberschenkels & 45 \\
\hline Am Trochanter . . . & 51 \\
\hline Am oberen Rand der Patella & 34 \\
\hline Am unteren Rand der Patella & 31 \\
\hline Mitte des Unterschenkels. . & 29 \\
\hline
\end{tabular}

Der rechte Ober- und Unterschenkel sind also ziemlich gleichmässig im Dickenwachsthum zurïckgeblieben. Der rechte Fuss ist eigenthümlich deformirt und zeigt ungefähr das Ausseben des Pes varus. Er ist beträchtlich kürzer als der linke, welcher vollkommen norrnal gebildet ist. Die Maasse ergeben :

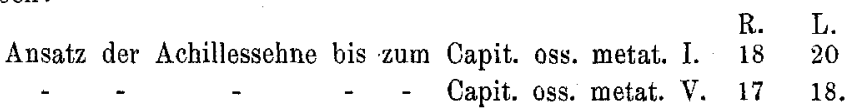

Die Circumferenz des Fusses in der Gegend des Lisfrane'schen Gelenkes beträgt beiderseits $25 \mathrm{~cm}$. Die rechte grosse Zebe ist dorsalwärts luxirt und bedeutend kleiner als die linke. Genaue Messung ist wegen der Stellungsanomalie nicht möglich. Die 2. Zehe ist ziemlich normal gebildet, aber kürzer als auf der anderen Seite.

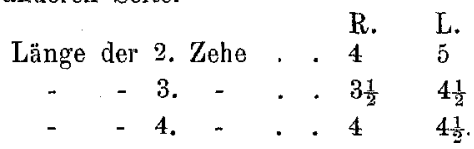

Die rechte 5. Zehe stellt nur einen kleinen, narbigen Stummel dar, der keinen Nagel mehr besitzt und mit der 4. Zehe vollständig verwachsen ist. Durch die Palpation constatirt man, dass dieser Stummel noch einen beweglichen Knochen, die Grundphalanx aufweist. Der rechte Kleinzehenballen ist vou einer dicken, schwieligen Haut bedeckt, die ausserdem von mehreren Narbensträngen durchzogen ist. In der Mitte dieser schwieligen Hautpartie, ungefähr der Gegend des Cap. metat. V entsprechend, befindet sich eine Fistelöffung, durch welche die Sonde $1 \frac{1}{2} \mathrm{~cm}$ tief eindringt, worauf sie auf blossliegenden Knochen stösst. Die Fistel secernirt ganz geringe Mengen dünnen Eiters. Auf dem Dorsum des rechten Fusses befindet sich ebenfalls eine Narbe, die angeblich nicht durch Stiefeldruck entstanden sein soll. In der rechten Inguinalgegend ist eine haselnussgrosse Lymphdrüse zu fühlen. Die rohe Kraft des rechten Beines ist kaum geringer, als diejenige des linken und sämmtliche Bewegungen desselben gehen sowohl activ als passiv leicht und sicher von statten; nur die Zehen können activ fast gar nicht bewegt werden, während dies passiv ganz leicht möglich ist. Die Sensibilität ist an Ober- und Unterschenkel beiderseits ziemlich gleich. Die Tastkreise sind auf beiden Seiten von derselben Grösse. Bedentend herabgesetzt ist dagegen die Sensibilität an der rechten Planta pedis, insbesondere in der unmittelbaren Umgebung der Fistel, woselbst eine Nadel tief eingestochen werden kann, ohne dass Patient etwas davon empfindet. Aber auch an der übrigen 
Planta pedis werden leichte Reize, die links ganz gut empfunden werden, nicht gefühlt. Die Tastkreise sind hier auf das Doppelte vergrössert. Hautreflexe werden rechts auf ganz kleine Reize ausgelöst und es bewegen sieh dabei die Zehen deutlich mit, obschon sie, wie oben bemerkt worden, activ gar nicht zu bewegen sind. Der Patellarreflex ist rechts fast aufgehoben, links ganz deutlich. Ein Temperaturunterschied zwischen beiden Extremitäten ist nicht zu constatiren, es fehlt auch jede abnorme Färbung der Haut am kranken Bein. Die elehtrische Untersuchung, die Herr Prof. Eichhorst vorzunehmen die Güte hatte, ergab Folgendes:

Es reagiren die Muskeln auf den faradischen Strom an beiden Extremitäten gleich. Die Prüfung mit dem constanten Strom ergiebt ebenfalls vollkommen normale Erregbarkeit. Bei 8 Elementen erfolgen die Ausschläge an den Extensoren des Unterschenkels beiderseits gleich kräftig und es ist keinerlei Abweichung rom normalen Zuckungsgesetz zu constatiren. Der Arterienpuls an Femoralis and Tibialis postica ist deutlich fühlbar; ein Unterschied in der Qualität ist nicht vorhanden. Die sphygmographische Curve, deren Aufnahme keine Schwierigkeiten macht, bietet an beiden Aa. femorales dasselbe Bild, welches in keiner Weise ron der bei anderen Individuen aufgenommenen Curve abweicht.

Betrachten wir den Rücken des Patienten, so fällt uns neben einer leichten dextro-convexen Skoliose der Brustwirbelsäule sofort auf, dass die Haut über der Gegend der Lendenwirbel in circumseripter Ausdehnung mit langen Haaren besetzt ist (s. Zeichnung). Die obere Grenze dieses Haarfeldes entspricht der Höbe des 1. Lendenwirbels und ist $28 \mathrm{~cm}$ vom After entfernt. Die Höhe desselben umfasst $4 \mathrm{~cm}$, die Ausdehnung nach der Seite hin je $8 \mathrm{~cm}$. Die Haare selbst sind bräunlich-blond und dünn; die grössten derselben, $10 \mathrm{~cm}$ lang, bilden Haarlocken, die 4 an der Zahl aus dem Centrum des Haarfeldes hervorwachsend, schief nach unten gerichtet sind und mit ihren Spitzen nach oben sich umbiegen. Nach den Seiten hin sind die Haare spärlicher und heller gefärbt, fast alle convergiren gegen die Mittellinie. Die Haarbüschel lassen sich leicht gegen den Kopf hin durch Streichen aufrichten, aber auch ebenso leicht gegen den Steiss za wieder niederlegen. In der Mittellinie wird, went die Haare zur Seite gebogen werden, eine ganz kleine, weisse Narbe sichtbar; sonst fehlt jede Verdickung oder abnorme Pigmentirung der Haut.

Beim Abtasten der Wirbelsäule in dieser Gegend geräth man unterbalb des ersten Lendenwirbels, statt dass man die bier sonst stark vorspringenden Processus spinosi fäblt, in eine flache Grube von $3 \mathrm{~cm}$ Höhe und $4 \mathrm{~cm}$ Breite, Druck daselbst verursacht keinen Schmerz, man stösst auch nicht anf federnden Widerstand, sondern fühlt im Grunde der Vertiefung eine feste Resistenz. Bei genanem Zuseben ist diese flache Grube anch sichtbar, namentich fällt sie dann auf, wenn man die Lendenwirbelsäule des Patienten mit derjenigen eines normal gebauten Individuums vergleicht. Die Processus spinosi des Kreuzbeins sind undeutlich fühlbar. Betrachten wir nochmals den ganzen Patienten, unsere Aufmerksamkeit auf die Entwicklung der Haare am übrigen 
Körper richtend, so bemerken wir, am Scheitel beginnend, dass die Kopfhaare dicht, hellbraun und nicht gelockt sind. Am Gesicht leicbter Kinnbart, an Hals, Brust, Bauch und Armen sehr wenige Haare; in der Axilla, am Mons veneris und in der Gesässfalte reiche aber nicht abnorme Entwicklung. Nirgends am Körper, ausser an der beschriebenen Stelle, vereinzelte Büschel. An den Nates sind ganz feine, spärliche, kaum sichtbare Härchen vorbanden, über deren Richtung nichts Besonderes auszusagen ist. Der Haarwuchs an der kranken Extremität ist sehr wenig entwickelt und keineswegs reichlicher, als am gesunden Bein.

Da die Ulceration am Fusse bei jeder anderen Behandlung nur vorübergehend zur Heilung gelangte, so wurde dem Patienten vorgeschlagen, den mit der Fistel behafteten Theil des Fusses entfernen zu lassen. Diese Operation wird am 2. Juli 1884 von Herrn Prof. Krönlein vorgenommen. Es werden in Narkose, unter Esmarch'scher Blutleere die Lappen zur Lisfrane'schen Exarticulation formirt. $D_{a}$ es sich jedoch nach Blosslegung des Gelenks zeigt, dass auch die Ossa cuneiformia stark destruirt sind, so wird die Amputation nach Bona ausgefübrt. Bei der Unterbindung der Gefässe wird eine auffallende Derbheit und Dicke der Arterienwandungen constatirt. Die Heilung erfolgte per primam int. und der Verlauf war ein fieberfreier. 5 Wochen nach der Operation konnte Patient mit schönem Stumpfe, an dem die Sensibilität vollkommen normal war, geheilt entlassen werden.

Leider fehlt aus dieser Zeit der pathologisch-anatomische Befund des Präparates.

Es verstrich ein Jahr, da kam Patient abermals in unsere Behandlung und zwar wieder wegen einer, der früheren ganz ähnlichen Fistel, die sich am Amputationsstumpfe ganz allmählich entwickelt hatte. Auf der äusseren Seite der Gehfläche des im Uebrigen schön gebildeten Stumpfes war eine Ulceration von $\frac{1}{4} \mathrm{~cm}$ Durchmesser vorhanden, deren Grund von blassen Granulationen ausgefüllt war. Die Sonde drang nicht auf blossliegenden Knochen, sondern nur anf starres Fasciengewebe. Um die Fistel berum überall schwielige Verdickung des Epithels und im Umkreis von $2 \mathrm{~cm}$ totale Anästhesie. Im Uebrigen der früher geschilderte Status praesens. Die Atrophie der kranken Extremität war nicht hochgradiger geworden. - Am 7. Juli 1886 wird von Herrn Prof. Krönlein die Amputation nach Pirog off ausgeführt. Eine Destruction der das Fussgelenk bildenden Knochen ist dabei nicht zu constatiren; es fehlt nur an einer Stelle der Talusrolle der knorplige Ueberzug. Die Blutung nach Abnahme der Esmarch'schen Binde ist auffallend gering. An den Gefässen zeigen sich wieder dieselben dicken Wandungen und kleinen Lumina. Zur Bedeckung des Stumpfes wird nur Haut mit vollkommen intacter Sensibilität verwendet.

Der Heilverlauf war ein gänzlich fieberfreier. Die Anbeilung des Knochenabschnittes des Calcaneus erfolgte langsam, doch kam knöcherne, solide Consolidation zu Stande. Patient konnte am 10. September $1886 \mathrm{mit}$ sehr scbönem Piroggff'schem Stumpfe, an dem keine Störung der Sensibilität vor banden war, entlassen werden. 
Der dnreh die Operation abgetragene Theil des Fusses wurde im pathologischen Institut von Hern Prof. Klebs untersuebt. Der uns zur Pablication gütigst überlassene Befund lautet folgendermaassen:

Ulcus perforans pedis, bei Spina bifida occulta, Neuritis hyperplastica. Der uns in frischem Zustande nach der Exarticulation übersendete Theil zeigte ausser einem trichterförmigen, an der Sohlenfläche vorhandenen Geschwür keine auffallenden Veränderungen, weshalb nur ein Theil der Weichtheile in-der Umgebung des Geschwürs zur Untersuchung aufbewahrt wurde. So ist es leider unterblieben, die Verhältnisse der Knochen genauer zu prüfen auf die Anwesenheit atrophischer Prozesse, welche sich an denselben in weiter vorgeschrittenen Fällen vorfinden. Besonders gross können dieselben nach dem äusseren Ansehn des Theils in diesem Falle nicht gewesen sein.

Der Fall ist deshalb von besonderer Wichtigkeit, weil er die erste Entstehung des sog. Mal perforant du pied deutlich erkennen lässt, indem neben einer grossen trichterförmigen Ulceration, etwa $1 \mathrm{~cm}$ von derselben entfernt; ein frischerer Erkrankungsheerd sich zeigte, an welchem sich unter einer intacten Epidermis von mässiger Verdickung schon makroskopisch eine stärker geröthete Granulationsgewebsmasse erkennen liess, welche zapfenartig in die Epidermisschicht eindrang. Die an gefärbten Schnitten des gehärteten Präparates angestellte mikroskopische Untersuchung ergab einen vollstïndig übereinstimmenden' Bau des jüngeren und älteren Heerdes. In beiden handelt es sich um eine Bildung von Granulationsgewebe, welche innerhalb des Bereichs einzelner Gefässbahnen sich vollzieht. In der gewöhnlichen Weise findet man zuerst längs der Gefässe eingelagerte Rundzellen, welche danu allmählich sich in spindelförmige Elemente umwandeln. Die derben Bündel des Cutisgewebes werden durch diese immer weiter sich verbreitende Neubildung durchsetzt und anseinandergedrängt, während der ganze Papillarkörper in eine zellreiche Masse verwandelt wird, welche von weiten, dünnwandigen und klaffenden Capillargefässen durchzogen und offenbar in ïbermässig reichlicher Weise ernährt wird. Wo die in Granulationsgewebe verwandelten Papillen in die Epidermis eindringen, zeigt auch die letztere eine der Hyperplasie der bindegewebigen Theile entsprechende Weiterentwickelung: die 
Epithellagen zwischen den Papillen werden breiter, als in den benachbarten Theilen, zeigen aber sonst keine Abweichung von dem Normalen. Kerntheilungsfiguren sind hier nicht mehr nachzuweisen.

An diesen hyperplastischen Vorgängen nehmen nun in ganz auffallender Weise auch die Wandungen der grösseren, namentlich arteriellen Gefässe theil. Ihr Lumen erscheint verengert, doch dürfte dies nur auf Rechnung der Contraction zu setzen sein; hie und da scheinen allerdings auch die Elemente der Intima vermehrt zu sein, indem die zellreichere Membran stark in das Lumen des Gefässes hineinragt. Im Allgemeinen indess überwiegt die Hyperplasie der Muskelschichten, welche durch eine auffallende Vergrösserung der musculären Elemente bedingt wird; Vermehrung derselben durch Theilungsvorgänge ist nicht nachzuweisen, freilich auch nicht auszuschliessen. - Man muss sich demnach den ganzen Vorgang als eine partielle Hyperplasie der Gewebe vorstellen, welche stets im Zusammenhange mit einer entsprechenden Hyperplasie der arteriellen Blutgefässe steht, entweder von der letzteren ausgeht oder als Coëffect einer und derselben Ursache betrachtet werden muss. Die Zerstörung der Gewebe und die Geschwürsbildung beruht nur auf den mechanischen Wirkungen der unter der derben Epidermis vor sich gehenden Granulationsbildung, wie auch auf der grösseren Hinfälligkeit des letzteren Gewebes. Vermittelt und eingeleitet wird diese Zerstörung sehr häufig durch Blutextravasate, welche sich in den hyperplastischen Papillen vorfinden und wohl auf mechanische Insulte zurückgeführt werden müssen.

Die fundamentale Frage in allen solchen, zur Nekrose führenden Granulationsgewebsbildungen betrifft nun die Beschaffenheit der nervösen Elemente. Bei dem vorliegenden Fall, welcher ganz mit den von v. Recklinghausen neuerdings beschriebenen Fällen von Spina bifida occulta und lumbaler Hypertrichose übereinstimmt, kann es wohl nicht fraglich sein, dass der angebornen Störung des Rückenmarks ein Einfluss auf das Zustandekommen der peripherischen, zur Nekrose führenden Granulationsbildung zuzuschreiben sei; doch besteht noch insofern ein $Z$ weifel, ob neben den angenommenen, in den v. Recklinghausen'schen Fällen auch nachgewiesenen Störungen am Rückemark Verände- 
rungen der peripherischen Nerven nachweisbar sind. Indem v. Recklinghausen dieselben leugnet, erwächst der Anschein, als ob hierin ein fundamentaler Unterschied zwischen diesen, durch eine centrale Störung bedingten neuropathischen Ulcerationen und dem gewöhnlichen Mal perforant du pied vorhanden wäre. Bei dem letzteren sollen, wie F. Fischer anführt, stets Veränderungen an den peripherischen Nerven vorhanden sein. Nach dem vorliegenden Fall scheint indess das Gleiche bei den centralen Formen neuropathischer Störungen vorzukommen, indem sehr deutliche Veränderungen an den peripherischen Nerven des amputirten Theils nachzuweisen sind. Die kleineren Nerven sind in dem gewucherten Hautgewebe nur schwer nachzuweisen; doch zeichnen sich manche der die derben Cutisfasern durchsetzenden zellreichen Züge durch ihren Reichthum an spindelförmigen Elementen aus, welche dichte, ziemlich scharf begrenzte Züge von paralleler Anordnung bilden. Die länglichen Kerne gehen oft in leicht wellige, feine Fasern über. Eine vollkommene Sicherheit, dass es sich hier um Nerven handelt, welche nur aus feinen Fasern bestehen, gewinnt man indess, wenn man die in Alkohol gehärteten Präparate in schwacher Essigsäure aufquellen lässt und die weiss bleibenden Züge isolirt. Man gewinnt so die Nervenstämmehen von 1 bis herab zu etwa $\frac{1}{4} \mathrm{~mm}$ und kann dieselben leicht von dem anhängenden Bindegewebe befreien. Bei schwacher Vergrösserung sieht man jetzt sehr deutlich die Zusammensetzung derselben aus feinen, gewellten, in Essigsäure nicht aufquellenden Fasern, in denen in spindelförmigen Anschwellungen längliche Kerne eingelagert sind. Von markhaltigen Fasern ist in diesen isolirten Stücken gewöhnlich nichts zu sehen. Aeusserlich werden dieselben ziemlich fest durch ein dünnes, aber resistentes Neurilem zusammengehalten. An der nerpösen Natur dieser Bildungen ist nicht zu zweifeln, doch ist das scheinbar gänzliche Fehlen markhaltiger Fasern auffallend. Erst nach dem Zerzupfen bemerkt man indess im Innern der Bündel regelmässig eine geringe Menge solcher, die aber sämmtlich, soweit ich gesehen, hochgradig degenerirt sind. Ihre Markhülle ist zerfallen und zum Theil durch ungleich grosse Fetttropfen. ersetzt, der Axencylinder nicht nachzuweisen. Auch 
Safraninfärbung hat mir in dieser Beziehung keine positiven Resultate ergeben.

Es handelt sich demnach um eine hyperplastische Neuritis mit reichlicher Neubildung markloser, den embryonalen Typus bewahrender Nervenfasern, während von den älteren markhaltigen Elementen nur wenige in gänzlich degenerirtem Zustande übrig geblieben sind. $O b$ diese Neubildungsvorgänge den degenerativen gefolgt sind, lässt sich aus dem Befunde leider nicht ermitteln, da die Verbreitung der beiden Veränderungen nach oben hin gegen das nervöse Centrum nicht weiter verfolgt werden konnte; doch ist diese Annahme wahrscheinlich; fraglich bleibt dagegen, in welcher Beziehung die Neubildung von jungen Nervenfasern zu den centralen, von der Spina bifida occulta abhängenden Störungen steht. Eine directe, Zellproliferation anregende Wirkung kommt, so weit wir wissen, keiner solchen Störung zu und bleibt deshalb auch hier, wie bei allen neuroparalytischen Zuständen nur die Wahl übrig zwischen einer Einwirkung peripherischer Reize auf den neurotisch geschwächten, namentlich insensiblen Theil, oder einem Ausfall von Hemmungsvorrichtungen, deren Wirkung sich auf die trophischen Zustände bezieht. Da Reizungen gelähmter Theile in der Regel nicht derartige elephantiastische Zustände herbeiführen, bin ich geneigt, der zweiten Hypothese den Vorzug zu geben. Es ist dieselbe um so annehmbarer, als in anderen Fällen von neuroparalytischen, mit Granulationsbildung beginnenden Geschwüren Reizungen der betroffenen Theile nicht annehmbar sind oder gänzlich innerhalb des Bereichs der gewöhnlichen Irritationen liegen, welche jeden oberflächlichen Theil treffen. In jedem Falle kommt solchen Reizungen, wenn man für dieselben noch diese Bezeichnung zulassen will, eine ganz nebensächliche Bedeutung zu und ist der gesteigerten formativen Irritabilität der Theile das Hauptgewicht beizulegen. Ich wäre geneigt, der nachgewiesenen hyperplastischen Neuritis einen entscheidenden Einfluss auf die hyperplastische Entwickelung der Gewebe zuzuschreiben und sie selbst wiederum abhängig zu machen von einem Ausfall centraler Hemmungsvorrichtungen; doch muss dahingestellt bleiben, ob und welche gangliösen Veränderungen die Entstehung der letzteren veranlassen. 
Es ist ersichtlich, dass diese Fragen tief eingreifen in die ganzo Lehre der pathologischen Neubildung und daher nur im Zusammenhang mit den Erscheinungen der letzteren Reihe behandelt werden können.

Zürich, 7. Oct. 1886.

Prof. Klebs.

Durchlesen wir die wenigen Krankengeschichten und Arbeiten, die über Spina bifida occulta publicirt worden sind und werfen wir einen Rückblick auf das von uns entworfene klinische Bild, so gelangen wir zunächst zu der Ansicht, dass bei unserem Falle die Diagnose insofern sicher steht, als keines jener Momente fehlt, durch welche nach Virchow das Wesen der Spina bifida occulta am Lebenden charakterisirt wird. Unter den 5 schon bekannten Fällen hat mit dem unsrigen am meisten Aehnlichkeit der von F. Fischer zuerst beschriebene Patient. Bei beiden Individuen ist das Haarfeld der Hypertrichose gleich localisirt und geformt, doch sind bei unserem Patienten die Haare mehr zu Büscheln gestellt und sie erreichen nicht dieselbe Länge. Die deutlich abtastbare abnorme Vertiefung des Rückens befindet sich hier wie dort im Bereiche der Lendenwirbelsäule und zwar reicht sie vom 1.-5. Proc. spinosus. Beide Patienten zeigen neben einer Difformität des Fusses, die wohl zurückzuführen ist auf eine congenitale Störung des Rückenmarks und der Nervenursprünge, jene mit Anästhesie der Haut einhergehende Ulceration der Planta pedis, die wir "mal perforant" zu nennen, keinen Anstand nehmen, da sowohl die klinischen Symptome als auch der pathologisch-anatomische Befund für diese Diagnose sprechen.

von Recklinghausen ist in der citirten Arbeit, gestützt auf die Beobachtung seines Falles zu dem Schlusse gekommen, dass die Anordnung des übermässigen Haarwuchses an der Lendenkreuzbeinhaut bei Spina bifida occulta demselben Gesetze folge, welches den Haarstrich in der Umgebung der offen liegenden Spina bifida beherrsche.

Die Haare der Steisshaut streichen physiologisch in horizontal gerichteten und alsdann nach unten gegen das Steissbein abgebogenen Linien. Bei dem von v. Recklinghausen secirten Patienten sind alle Haare am Gesäss mit ihrer Spitze gegen das 
Centrum der Spina bifida gerichtet und bilden hier einen convergirenden Wirbel. Diesem, auf der Lendenkreuzbeinhaut gelegenen Haarwirbel mit seiner Umkehr des sacralen Haarstromes legt v. Recklinghausen für die Diagnose der Spina bifida occulta eine besondere Bedeutung bei. Eine Bestätigung dieses Satzes bietet unser Fall deshalb nicht, weil die Haut über dem Kreuzbein, an welcher nach v. Recklinghausen diese typische Umkehr des Haarstriches hervortreten muss, haarfrei ist. Es ist aber nicht nur die Aenderung der Haarstellung, sondern auch eine ausgesprochene Büschelbildung der Haare, die nach v. Recklinghausen die genetische Beziehung zwischen der Hypertrichosis sacralis und der Spina bifida illustrirt und in diesem Punkte stimmt unser Fall mit seiner Beobachtung vollkommen überein, indem, wie unsere Abbildung zeigt, im Centrum der Hypertrichose die Haare in Büscheln gruppirt sind, während sie nach den Seiten hin einzeln hervorwachsen. Es ist möglich, dass auch bei unserem Patienten unter der Hypertrichose im Wirbelkanal eine geschwulstartige Wucherung des Fett- und Muskelgewebes stattgefunden hat; in vivo ist dies nicht zu eruiren. Eine Bestätigung. der v. Recklinghausen'schen Erklärung der vermehrten localen Haarbildung durch die den Tumoren zu Grunde liegenden Hyperplasien kann also unser Fall vorläufig nicht bieten. 